\title{
The effect of Laparoscopic Sleeve Gastrectomy on Obstructive Sleep Apnea Syndrome
}

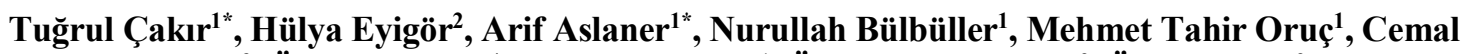 \\ Polat $^{3}$, Özgür Balasar ${ }^{4}$, Himmet Durgut ${ }^{1}$, Ömer Tarık Selçuk ${ }^{2}$, Üstün Osma ${ }^{2}$ \\ ${ }^{1}$ Department of General Surgery, Antalya Training and Research Hospital, Antalya, Turkey \\ ${ }^{2}$ Department of Ear Nose Throat, Antalya Training and Research Hospital, Antalya, Turkey \\ ${ }^{3}$ Department of Biochemistry, Public Health Laboratory, Kütahya, Turkey \\ ${ }^{4}$ Department of Genetics, Antalya Training and Research Hospital, Antalya, Turkey
}

\begin{abstract}
Background: The repeated episode of obstructive hypopnea and apnea during sleep is defined as obstructive sleep apnea (OSA) and it is a common condition in obese patients. Studies performing bariatric surgery have demonstrated a significant improvement in OSA by weight reduction. In this prospective study we aimed to explore the efficacy and safety of Laparoscopic Sleeve Gastrectomy (LSG) on OSAS among severely obese patients. Material and Methods: A total of 32 morbidly obese patients who underwent LSG for morbid obesity were included in this study. Body weight, height, body mass index (BMI) and standard overnight polysomnography (PSG) were measured at before and after LSG at the 6th month. Results: 32 patients (27 female, 5 male) who have postoperative $P S G$ 's were included in this study. The mean age was $43.22 \pm 9.87$ years old. The mean preoperative and postoperative BMIs were $50.36 \pm 8.14 \mathrm{~kg} / \mathrm{m}^{2}$ and $37.27 \pm 7.93 \mathrm{~kg} / \mathrm{m}^{2}$, respectively. The mean Epworth sleepiness scale determined as $5.84 \pm 4.65$ preoperatively and $2.19 \pm 3.55$ postoperatively. The preoperative and postoperative sleep efficiency test of the patients was determined as $83.34 \pm 9.68$ and $88.94 \pm 6.90$ respectively. AHI average at the preoperative $P S G$ was $31.47 \pm 26.34$, while 9.35 10:34 at postoperative 6 months and found as statistically significant. Conclusion: Our data showed that LSG is an efficient and safe procedure on severely obese patients and showed a predictive remission of clinical and sleep parameters of patients with OSA by analyzing PSG data during the first 6 months.
\end{abstract}

Key words: Laparoscopic sleeve gastrectomy; OSAS; polysomnography

\section{Corresponding Author:}

Tuğrul Çakır $^{1^{*}}$, Arif Aslaner ${ }^{*}$

Varlik Mahallesi, Kazım Karabekir Caddesi Soğuksu, Antalya, 07100, Turkey

Email address: arifaslaner@gmail.com

\section{Introduction}

Recurrent episode of hypopnea or apnea during sleep is known as obstructive sleep apnea (OSA) (1). Obesity is one of the risc factor of this multifactorial disease (3). The prevalence of OSA range from 60$83 \%$ in patients planned for bariatric surgery (4). Nowadays popular bariatric surgery; Laparoscopic Sleeve Gastrectomy (LSG) is commonly performed because of its positive results on body weight loss and having less comorbidities (5). Some studies reported that after bariatric surgery patients had a high rate of improvement or resolution of obesity associated comorbidities such as diabetes, hypertension, hyperlipidemia and OSA (4,6-9). Studies after LSG based on polysomnography (PSG) for the evaluation of OSA are needed.

In this present study, we aimed to evaluate the efficiency of LSG by losing weight during the first 6 months for treating OSA by analyzing PSG findings obtained before and after surgery in morbidly obese patients

\section{Material and Methods}

Study design and patients:

This study was performed at the department of general surgery of Antalya Education and Research Hospital and the clinical evaluation were performed at the Sleep Laboratory of the Ear Nose Throat Department. The consent of the Ethics Committee at Antalya Education and Research Hospital (2013/117) was achieved and additional informed consent was obtained from all individual participants for whom identifying information is included in this article. All procedures performed in studies involving human participants were in accordance with the ethical standards of the institutional and/or national research committee and with the 1964 Helsinki declaration and its later amendments or comparable ethical standards. Body weight and height were measured and Body Mass Index (BMI) was calculated. The following BMI grading system was implemented: normal range (BMI, 18.5-24.9kg/m2), overweight 
(BMI, 25-29.9kg/m2), obese (BMI, 30-34.9 kg/m2), severely obese (BMI, 35-39.9kg/m2), morbid obese (BMI, 40-49.9kg/m2), super obese (BMI, >50 $\mathrm{kg} / \mathrm{m} 2$ ). A total of 32 morbidly obese patients (5 male, 27 female), aged 19-62 years with a BMI $\geq 40$ $\mathrm{kg} / \mathrm{m} 2$, were operated on from July 2013 to February 2014. Follow-up inspections were carried out at the 6th month. Body weight, height, BMI and standard overnight PSG were measured before the operation and at the 6th month.

Surgical Procedure:

All patients underwent standard five port laparoscopic sleeve gastrectomy by the same surgeon. It was made by Echelon 60 (EthiconMexico) linear stapler. Postoperative care was uneventful and managed in the same manner.

Polysomnography Analysis:

Patients suspected of narcolepsy, hypersomnolence, periodic limb movement disorders were excluded from the study. Patients with psychiatric or neurological disorders and major systemic co-morbidities were excluded. Epworth Sleepiness Scale (ESS) score was assessed for each patient using the validated Turkish version of the ESS questionnaire (10). The ESS questionnaire assesses the general level of daytime sleepiness by having individuals rate the likelihood of dozing during eight different daytime situations (scores 0 10). Body Mass Index (BMI) was calculated as weight (kg) divided by the height-squared $(\mathrm{m} 2)$.

The diagnosis of OSA was established by a Polysomnography (PSG) attended overnight (Grasstelefactor - PMA AS40) in the sleep laboratory of our department. Measured parameters included electroencephalography (C4/A1, O2/A1, F4/A1, F3/A2), electro-oculography, electrocardiogram, oronasal airflow either by nasal cannula or thermal sensors, pulse oximetry, thoracoabdominal movements, submental and pretibial electromyography and snoring noises.

Obstructive apneas were defined as cessation of airflow for at least $10 \mathrm{~s}$. Hypopneas were defined as periods of reduction of $>30 \%$ oronasal airflow for at least $10 \mathrm{~s}$ and $\mathrm{a} \geq 4 \%$ decrease in oxygen saturation. PSG data were manually scored according to the recommendations of the American Academy of Sleep by the same sleep technician under the supervision of an attending sleep specialist (11). An Apnea Hypoapnea Index (AHI) of $5-15 / \mathrm{h}$ is mild OSAS, an AHI of $15-30 / \mathrm{h}$ is moderate and AHI $>30 / \mathrm{h}$ is severe OSAS, as assessed by polysomnography (12).

Statistical Analysis

The statistical analysis of the data was performed by using the SPSS 15.0 (SPSS Inc., Chicago, IL, USA) package program and Microsoft Office Excel version 2010. To comply with data of normal distribution One-Sample Kolmogorov-Smirnov test was used. In the comparison of data Mann-Whitney $\mathrm{U}$ test, Paired Samples Test, Wilcoxon test,
Spearman's and Pearson's correlation tests were used. For the descriptive statistical analysis of the data: mean \pm standard deviation and minimum-maximum values for numeric variables; numbers and percentages for categorical data were used. The level of $\mathrm{p}<0.05$ was considered as significant.

\section{Results}

57 of 67 patients who planned bariatric surgery with a total of AHI $>5$ revealed and preoperative PSG testing was performed. However, 32 patients who have postoperative PSG's were included in this study. 27 of these patients were female $(84 \%)$ and 5 were male $(16 \%)$. The mean age of the patients was $43.22 \pm 9.87(21-61)$ years old. Demographic characteristics of the patients are shown in Table 1 .

The mean preoperative BMI was $50.36 \pm 8.14$ (38.71-74.04) $\mathrm{kg} / \mathrm{m}^{2}$, whereas the mean postoperative BMI was $37.27 \pm 7.93(24.22-53.28) \mathrm{kg} / \mathrm{m}^{2}$ respectively. The mean Epworth sleepiness scale (ESS) determined as $5.84 \pm 4.65$ preoperatively and $2: 19 \pm 3: 55$ postoperatively. Only 1 of the 8 patients defining excessive daytime sleepiness (Epworth sleepiness scale $>10$ points) preoperatively had excessive daytime sleepiness postoperatively. The rate of patients with comorbid diseases (hypertension, diabetes, COPD) was $31 \%$. The preoperative and postoperative sleep efficiency test of the patients was determined as $83.34 \pm 9.68$ and $88.94 \pm 6.90$ respectively.

AHI average at the preoperative PSG was $31.47 \pm$ 26.34 (63.50-97.00), while $9.35 \pm 10: 34$ (0.50-43.40) at postoperative 6 months and found as statistically significant $(p<0.001) .40 .625 \% \quad(n=13)$ of the patients included in this study have been identified as mild OSAS, $18.75 \%(n=6)$ moderate OSAS and $40.625 \%(\mathrm{n}=13)$ severe OSAS. The postoperative AHI was detected under $<5$ in $16(16 \%)$ patients, while it was found between 5-15 in 10 patients, between $15-30$ in 3 patients and over $>30$ in 3 patients. The preoperative and postoperative PSG findings of the patients are summarized in Table 2.

In the comparison of preoperative BMI and PSG findings, moderate positive correlation between nonsupine AHI and REM AHI $(r=0.546, r=0.536)$, moderate negative correlation between the preoperative low oxygen saturation $(\mathrm{r}=-0.549)$ were found (Table 3 ). There was no significant correlation found at the comparison of postoperative BMI and PSG parameters (Table 4). 
Table 1:

\begin{tabular}{|c|c|c|c|c|c|c|c|c|}
\hline & \multicolumn{4}{|c|}{ preoperative } & \multicolumn{4}{|c|}{ Postoperative 6 months } \\
\hline & $n$ & Mean & $S D$ & Range & $n$ & Mean & $S D$ & Range \\
\hline Age & 32 & 43.22 & 9.87 & $(21-61)$ & 32 & 43.22 & 9.87 & $(21-61)$ \\
\hline Weight(kg) & 32 & 130.75 & 17.90 & $(93-185)$ & 32 & 98.38 & 18.42 & $(68-128)$ \\
\hline$B M I\left(\mathrm{~kg} / \mathrm{m}^{2}\right)$ & 32 & 50.36 & 8.14 & $(38.71-74.04)$ & 32 & 37.27 & 7.93 & $(24.22-53.28)$ \\
\hline$E S S$ & 32 & 5.84 & 4.65 & $(0-15)$ & 32 & 2.19 & 3.55 & $(0-11)$ \\
\hline
\end{tabular}

Table 1: Preoperative and postoperative 6 months demographic characteristics of the patients

Table 2:

\begin{tabular}{|l|l|c|c|}
\hline & $\begin{array}{l}\text { Before surgery } \\
\text { Mean (SD) }\end{array}$ & $\begin{array}{c}6 \\
\text { surgery } \\
\text { Mean (SD) }\end{array}$ & P value \\
\hline Sleep efficiency (\%) & $83.34(9.68)$ & $88.94(6.90)$ & $<0.001$ \\
\hline AHI & $31.47(26.34)$ & $9.35(10.49)$ & $<0.001$ \\
\hline Minimum SaO, (\%) & $77.75(8.70)$ & $83.94(8.34)$ & $<0.001$ \\
\hline Supin AHI & $32.92(29.18)$ & $10.57(10.94)$ & $<0.001$ \\
\hline Nonsupin AHI & $28.39(31.46)$ & $6.36(11.89)$ & $<0.001$ \\
\hline REM AHI & $49.00(36.93)$ & $18.59(19.10)$ & $<0.001$ \\
\hline NonREM AHI & $27.29(28.07)$ & $7.38(9.18)$ & $<0.001$ \\
\hline
\end{tabular}

Table 2: Polysomnographic parameters before and 6 months after surgery

Table 3:

\begin{tabular}{|c|c|c|}
\hline \multirow{4}{*}{$\begin{array}{c}\text { Preoperative } \\
\text { MI }\end{array}$} & $\begin{array}{c}\text { Preoperative } \\
\text { PSG findings }\end{array}$ & $r=$ \\
\cline { 2 - 3 } & $\begin{array}{c}\text { Sleep } \\
\text { efficiency }\end{array}$ & 0.125 \\
\cline { 2 - 3 } & ESS & 0.142 \\
\cline { 2 - 3 } & AHI & 0.480 \\
\cline { 2 - 3 } & Supin AHI & 0.354 \\
\cline { 2 - 3 } & $\begin{array}{c}\text { Nonsupin } \\
\text { AHI }\end{array}$ & 0.546 \\
\hline REM AHI & 0.536 \\
\cline { 2 - 3 } & $\begin{array}{c}\text { NonREM } \\
\text { AHI }\end{array}$ & 0.436 \\
\cline { 2 - 3 } & $\begin{array}{c}\text { Minimum } \\
\text { SaO }\end{array}$ & -0.549 \\
\hline
\end{tabular}

Table 3: The relationship between preoperative PSG findings with preoperative BMI

Table 4:

\begin{tabular}{|c|c|c|}
\hline $\begin{array}{c}\text { Postoperative } \\
\text { months BMI }\end{array}$ & $\begin{array}{c}\text { Postoperative } \\
\text { 6 months } \\
\text { PSG findings }\end{array}$ & $r=$ \\
\cline { 2 - 3 } & $\begin{array}{c}\text { Sleep } \\
\text { efficiency }\end{array}$ & 0.275 \\
\cline { 2 - 3 } & ESS & 0.154 \\
\cline { 2 - 3 } & AHI & 0.367 \\
\hline Supin AHI & 0.153 \\
\hline & $\begin{array}{c}\text { Nonsupin } \\
\text { AHI }\end{array}$ & 0.117 \\
\cline { 2 - 3 } & REM AHI & 0.372 \\
\cline { 2 - 3 } & NonREM AHI & 0.348 \\
\cline { 2 - 3 } & $\begin{array}{c}\text { Minimum } \\
\text { SaO }\end{array}$ & -0.184 \\
\hline
\end{tabular}

Table 4: The relationship between postoperative 6 months PSG findings with postoperative 6 months BMI 


\section{Discussion}

Population based studies demonstrate OSAS affecting approximately $4 \%$ of males and $2 \%$ females (13). OSAS significantly increases the risk of stroke or death from any cause and in a community based sample moderate-to-severe sleep apnea is independently associated with a large increased risk of all-cause mortality. The obesity epidemic means problem faced by health professionals in relation to OSAS is only likely to increase in the immediate future (14).

Nowadays obesity is considered as one of the major risk factors for OSAS. The OSA rate is reported as $60-83 \%$ in patients with $\mathrm{BMI} \geq 35 \mathrm{~kg} / \mathrm{m} 2$ and scheduled for bariatric surgery. $(4,15)$. In our study, the prevalence of OSA was found to be $85.07 \%$ in patients with we have planned bariatric surgery. The higher rate of OSA may be associated with higher preoperative BMI $(50.36 \pm 8.14$ (38.7174.04)).

Surgical weight loss resulted in the significant improvement of OSA in obese individuals. As a result of the existing meta-analysis, data about the decline or improvement of OSA after bariatric surgery is available $(4,6,16)$.

In one recent meta-analysis, it was suggested that in 359 subjects with a mean BMI of $55 \mathrm{~kg} / \mathrm{m} 2$, surgery offered a weighted decrease of BMI by 16 $\mathrm{kg} / \mathrm{m} 2$ (16.1, $95 \%$ CI 13.3 to 18.9$)$ and a weighted decrease of AHI by 34 events/h (34.2, $95 \%$ CI 25.3 to 43.2) (4).

The first study reported at the 1980s and they defined a significantly decrease in the ratio of AHI after bariatric surgery. However in the study published in 1987, Charuz et al. reported a decrease of AHI from 58.8 to 36.1 with a weight loss of $70 \%$ at the 6 months after bariatric surgery (17). ValenciaFlores at al. reported the loss of OSAS and an improvement of oxygen saturation in $46 \%$ of patients having a decrease at BMI from 56.5 to $39.2 \mathrm{~kg} / \mathrm{m} 2$ after bariatric surgery (18).

Dixon at al. in a study with more complete follow-up, observed after 17 months a reduction in excess weight of $50 \pm 15 \%$ and a decrease in AHI from $61.6 \pm 34$ to $13.4 \pm 13$ events/hour, in addition to a large improvement in quality of sleep, in diurnal somnolence and in quality of life (19).

In our study, although early postoperative period (6 months), $50 \%$ of patients had postoperative AHI under 5 . The preoperative mean AHI $(30.72 \pm 26.43)$ was decreased to $10: 49 \pm 9: 27$ postoperatively and were found statistically significant $(\mathrm{p}<0.001)$.

It is of key importance to educate patients prior to bariatric surgery on OSAS: the high prevalence of OSAS in the bariatric surgery population, the risks of untreated OSAS, and need for appropriate treatment, especially since the effect of bariatric surgery on the AHI is not predictable. Ravesloot et al. have evaluated the postoperative PSG results with OSAS in two stages on patients they performed bariatric surgery. They reported a dramatically improvement on sleep parameters at the 7 th months and a slower recovery in the coming months (20).

Various retrospective cohort studies in bariatric surgery show potential benefit in the treatment of OSA yet may not be termed curative. A key focus is required on meaningful long-term weight loss maintenance following surgery $(21,22)$.

Bae et al. reported on bariatric surgery patients that postoperative residual OSA have more frequently seen in patients with preoperative low oxygen saturation and high preoperative supine AHI (23).

In our study, we observed high REM AHI in 11 $(68.75 \%)$ of 16 patients with residual OSA (AHI $>5)$. In our study it was also observed that the patients with residual OSA have preoperative low oxygen saturation (mean $73.75 \%$ ).

\section{Conclusions}

In conclusion, our study have been showed that LSG is an efficient and safe procedure on severely obese patients and showed a predictive remission of clinical and sleep parameters of patients with OSAS by analyzing PSG during the first 6 months.

\section{Acknowledgements}

Hicran Cantaş; technician at the sleep laboratory

\section{References}

1. Jordan AS, McSharry DG, Malhotra A. 2013. Adult obstructive sleep apnoea. Lancet. DOI:10.1016/S0140-6736(13)60734-5

2. Fritscher LG,Mottin CC, Canani S, Chatkin JM. 2007. Obesity and obstructive sleep apneahypopnea syndrome:the impact of bariatric surgery. Obesity surgery 17:95-99

3. Tishler PV, Larkin EK, Schluchter MD, Redline S. 2003. Incidence of sleep-disordered breathing in an urban adult population: the relative importance of risk factors in the development of sleep-disordered breathing. JAMA J Am Med Assoc 28917:2230-2237

4. Ashrafian H, le Roux CW, Rowland SP, Ali M, Cummin AR, Darzi A, Athanasiou T. 2012. Metabolic surgery and obstructive sleep apnoea: the protective effects of bariatric procedures. Thorax 67:442-449

5. Ren CJ, Patterson E, Gagner M. 2000. Early results of laparoscopic biliopancreatic diversion with duodenal switch: a case series of 40 consecutive patients. Obes Surg 10:514-523

6. Greenburg DL, Lettieri CJ, Eliasson AH. 2009. Effects of surgical weight loss on measures of obstructive sleep apnea: a meta-analysis. Am J Med 122:535-542 
7. Silecchia G, Boru C, Pecchia A, Rizzello M, Casella G, Leonetti F, Basso N. 2006. Effectiveness of laparoscopic sleeve gastrectomy (first stage of biliopancreatic diversion with duodenal switch) on co-morbidities in super-obese high-risk patients. Obes Surg 16:1138-1144

8. Cottam D, Qureshi FG, Mattar SG, Sharma S, Holover S, Bonanomi G, Ramanathan R, Schauer P. 2006. Laparoscopic sleeve gastrectomy as an initial weight-loss procedure for high-risk patients with morbid obesity. Surg Endosc 20:859-863

9. Moon Han S, Kim WW, Oh JH. 2005. Results of laparoscopic sleeve gastrectomy (LSG) at 1 year in morbidly obese Korean patients. Obes Surg 15:1469-1475

10. Izci B, Ardic S, Firat H, Sahin A, Altinors M, Karacan I. 2008. Reliability and validity studies of the Turkish version of the epwort sleepiness scale. Sleep Breath 12:161-168.

11. Berry RB, Budhiraja R, Gottlieb DJ, Gozal D, Iber C, Kapur VK, Marcus CL, Mehra R, Parthasarathy S, Quan SF, Redline S, Strohl KP, Davidson Ward SL, Tangredi MM. 2012. Rules for scoring respiratory events in sleep: update of the 2007 AASM Manual for the scoring of sleep and associated events. Deliberations of the Sleep Apnea Definitions Task Force of the American Academy of Sleep Medicine. J Clin Sleep Med JCSM Off Publ Am Acad Sleep Med 8:597-619

12. American Academy of Sleep Medicine. 2005. International Classification of Sleep disorders: Diagnostic and Coding manual,2nd edn., Westchester, IL

13. Lee W., Nagubadi S, Kryger MH, Mokhlesi B. 2008. Epidemiology of obstructive sleep apnea: a population- based perspective. Expert Rev Respir Med 1:349-364

14. Yaggi HK, Concato J, Kernan WN, Lichtman JH, Brass LM., Mohsenin V. 2005. Obstructive Sleep apnoea as arisk factor stroke and death. $\mathrm{N}$ Engl J Med 353:2034-2041

15. Dixon JB, Schachter LM, O'Brien PE. 2003. Predicting sleep apnea and excessive day sleepiness in the severely obese: indicators for polysomnography. Chest 123:1134-1141

16. Dixon JB, Schachter LM, O'Brien PE, Jones K, Grima M, Lambert G, Brown W, Bailey M, Naughton MT. 2012. Surgical vs conventional therapy for weight loss treatment of obstructive sleep apnea: a randomized controlled trial. JAMA J Am Med Assoc 308:1142-1149

17. Charuzi I, Fraser D, Peiser E et al. 1987. Sleep apnea syndrome in the morbidly obese undergoing bariatric surgery. Gastroenterol Clin North Am 16:517-519

18. Valerncia-Flores M, Orea A, Herrera $M$ et al. 2004. Effect of bariatric surgery on obstructive sleep apnea and hypopnea syndrome, electrocardiogram and pulmonary arterial pressure. Obes Surg 14:755762
19. Dixon JB, Schachter LM, O'Brien PE. 2005. Polysomnography before and after weight loss in obese patients with severe sleep apnea. Int J Obes 29:1048-1054

20. Ravesloot MJL, Hilgevoord AAJ, vanWagensveld B.A, N. de Vries. 2014. Assessment of the effect of bariatric surgery on obstructive sleep apnea at two postoperative intervals. Obes Surg 24:22-31

21. Lettieri CJ, Eliasson AH, Greenburg DL 2008. Persistence of obstructive sleep apnea after surgical weight loss. J Clin sleep Med JCSM .Off Publ American Acad Sleep Med 4:333-338

22. Kotecha BT, Hall AC. Role of surgery in adult obstructive sleep apnoea. 2014. Sleep Medicine Reviews 18:405-413

23. Bae EK, Lee YJ, Yun CH, Heo Y. 2014. Effects of surgical weight loss for treating obstructive sleep apnea. Sleep Breath 18:901-905 